\title{
Role of Fibroscan and Non Invasive Markers to Assess Hepatic Fibrosis and Steatosis at Initial Presentation of Patients with Hepatitis B
}

\author{
Ankur Shah" ${ }^{1}$, Rathi Chetan ${ }^{2}$ and Shah Jayshri A $^{3 *}$ \\ ${ }^{1}$ Consultant Surgeon, East Kent University Hospitals NHS Foundation Trust, \\ Ashford, UK and Director, Ansh Liver Clinic, Andheri West, Mumbai, India \\ ${ }^{2}$ Department of Gastroenterology, Jagjivan Ram Hospital, Western Railways, \\ Mumbai Central, Maharashtra, India \\ ${ }^{3}$ Consultant Gastroenterologist and Hepatologist, East Kent University Hospitals \\ NHS Foundation Trust, Ashford, UK and Director, Ansh Liver Clinic, Andheri West, \\ Mumbai, India \\ *Corresponding Author: Shah Jayshri A, Consultant Gastroenterologist and \\ Hepatologist, East Kent University Hospitals NHS Foundation Trust, Ashford, UK and \\ Director, Ansh Liver Clinic, Andheri West, Mumbai, India.
}

\begin{abstract}
Objectives: To analyse the Role of Fibroscan (FS) and non-invasive markers to assess hepatic fibrosis and steatosis at initial presentation of patients with Hepatitis B.

Methods: An observational prospective study of patients with chronic hepatitis B (CHB) evaluated at single Liver Clinic, Mumbai from April 2014-March 2017. Serological markers, transient elastography (fibroscan) for HF, APRI, FIB-4, AST/ALT ratio and E-score were analyzed. Controlled attenuation parameter (CAP) score on fibroscan was used for grading of hepatic steatosis. Patients were categorized into 2 groups: No significant fibrosis (< F2), significant fibrosis ( $>$ F2) group. AST/ALT ratio was divided into 2 groups: No significant fibrosis for $\leq 1$, significant fibrosis $>1$.

Results: 178 study patients with male preponderance (68\%), had asymptomatic infection, 24 patients were symptomatic; ascites (7), variceal bleeding (11) and hepatocellular carcinoma (8). 139 patients underwent fibroscan, 80 had HS on CAP score. 40/100 patients with normal AST and ALT had significant fibrosis ( $>$ F2) on fibroscan. Amongst noninvasive biochemical tools, only FIB-4 had significant correlation with fibroscan, $(\mathrm{p}<0.05)$. The ROC curve areas (AUROC) of FIB-4, APRI and AST/ALT ratio that differentiated patients with significant HF from without fibrosis was 0.704, 0.674, 0.567, respectively. The sensitivity and specificity of FIB-4, APRI and AST/ALT ratio to differentiate patients with significant HF from those without was $42.6 \%$ and $92.3 \%, 72.1 \%$ and $60.2 \%, 29.5 \%$ and $83.3 \%$, respectively.
\end{abstract}

Conclusion: Fibroscan and FIB-4 had significant correlation of HS in CHB patients. These can be used as non-invasive modalities to monitor HS in CHB patients.

Keywords: Hepatitis B; Hepatic Fibrosis; Hepatic Steatosis; Transient Elastography

Citation: Shah Jayshri A., et al. "Role of Fibroscan and Non Invasive Markers to Assess Hepatic Fibrosis and Steatosis at Initial Presentation of Patients with Hepatitis B”. Acta Scientific Gastrointestinal Disorders 3.8 (2020): 16-23. 


\section{Introduction}

Over half a billion of the world's population is chronically infected with hepatitis B (CHB), with approximately 35 to 45 million carriers in India alone [1]. Majority of patients infected with hepatitis B will remain as inactive carriers, however, a proportion of these patients will progress to cirrhosis and hepatocellular carcinoma (HCC). Approximately 2\% patients with hepatitis B will develop cirrhosis each year with a 100-fold increase in the risk of HCC in patients with hepatitis B compared to those without the infection. The development of cirrhosis and HCC is closely related to the severity of the underlying disease $[2,3]$. It is therefore critical to identify which factors contribute to accelerated liver injury and also to assess the stage of liver damage at the time of presentation, so that timely introduction of antiviral treatment can help reverse the fibrosis [4-6].

With rising incidence of non-alcoholic fatty liver disease (NAFLD), there will be more patients having hepatic steatosis coexisting with hepatitis B $[7,8]$. Traditionally ultrasound (USG) has been used to assess and grade steatosis. More recently controlled attenuation parameter (CAP) using the signals acquired by the fibroscan (R) has been developed as a method of assessing steatosis [9-11].

Noninvasive tools are being increasingly used in clinical practice to assess degree of fibrosis as well as monitor patient's response to therapy. Noninvasive tests include, serological markers, biological markers and imaging techniques [12,13]. Serological markers such as fibrosis index based on 4 factors (FIB-4), aspartate aminotransferase to platelet ratio (APRI), aspartate aminotransferase to alanine aminotransferase (AST/ALT) ratio are inexpensive and simple, as they incorporate routine laboratory results that are readily available. Recently, a meta-analysis reported that APRI and FIB-4 possess moderate diagnostic accuracy for predicting fibrosis in patients with CHB viral infection [14-16]. Numerous studies have been conducted to assess diagnostic accuracy of imaging tools for noninvasive assessment of fibrosis including fibroscan, magnetic resonance elastography (MRE), and acoustic radiofrequency imaging (ARFI) [17-20]. Some studies have used a combination of serological markers with imaging modalities for assessing the degree of fibrosis [21-26]. Liver biopsy, although the gold standard for steatosis and fibrosis assessment, has its own limitations. It is an invasive procedure with risk of complications which can be fatal, small tissue sample with inter and intraobserver variability limits its clinical application in all individuals $[27,28]$.

\section{Aim of the Study}

The aim of this study was to analyze the role of Fibroscan (FS) and non-invasive markers to assess hepatic fibrosis and steatosis at initial presentation of patients with Hepatitis B.

\section{Materials and Methods}

Inclusion and exclusion criteria: This is an observational prospective study of patients with chronic Hepatitis $B$, referred to Ansh Liver Clinic from April 2014 till March 2017.

\section{Exclusion criteria}

The following patients were excluded from the study (Seven categories). Patients found to have concomitant:

- Alcohol related liver disease - defined by alcohol intake exceeding $40 \mathrm{~g} / \mathrm{d}$ in males and $20 \mathrm{~g} / \mathrm{d}$ in females over the past 5 years

- Hepatitis B and C co-infection

- Hepatitis B and HIV or HCV co-infection

- $\quad$ Drug induced hepatitis

- Genetic or metabolic disease

- Autoimmune hepatitis

- $\quad$ Acute hepatitis B.

During this period, a total of 188 patients were referred with chronic hepatitis B, but 10 patients were excluded after application of above criteria.

Clinical characteristics: Data was recorded on those who had symptoms due to underlying hepatitis B infection as well as those who were asymptomatic, whereby the hepatitis B infection was detected incidentally. In the 178 patients with chronic hepatitis $B$ (CHB) data was collected on laboratory parameters including AST, ALT, platelet count, HBV DNA viral load, HbeAg status.

Data was collected on serological markers and fibroscan for fibrosis: APRI, FIB-4 and E-score.

APRI and FIB-4 were calculated based on the formula:

APRI $=[$ AST / ULN $] /$ Platelet count (10\%/L) [29-31]

FIB4 $=[($ age in years $) \times$ AST $(\mathrm{U} / \mathrm{L})] /\left[\right.$ Platelet count $\left(10^{9} / \mathrm{L}\right) \times$ $\sqrt{\text { ALT }(U / L)] ~}$

The upper limit for AST was $40 \mathrm{U} / \mathrm{L}$ [32]. 
Liver stiffness and CAP measurements: Liver stiffness measurement (LSM) and CAP was measured in 139 patients using Fibroscan (Echosens, France) M probe was used in 128 patients, 11 patient's required XL probe. Only results with 10 valid measurements and interquartile range (IQR)/median liver stiffness ratio $<30 \%$ were considered reliable. Both LSM measurements were obtained in the same area of liver parenchyma. The final LSM result corresponds to median LSM value expressed in KPa. Patients were categorized into 3 groups based on fibrosis score: Group I (<F2), Group II (F2-F3) and Group III (F4) as shown in table 1. For statistical analysis patients were categorized into 2 groups as: No significant fibrosis (< F2) and Significant fibrosis (> F2) group. Similarly, AST/ALT ratio was divided into 2 groups: No significant fibrosis group for value $<=1$ and significant fibrosis group for value $>1[33,34]$.

\begin{tabular}{|l|c|c|c|}
\hline Category & APRI value & FIB-4 & $\begin{array}{c}\text { Fibroscan E } \\
\text { score KPa }\end{array}$ \\
\hline$<$ F2 & $<0.7$ & $<1.45$ & $<7.2$ \\
\hline F2-F3 & $>0.7$ & $1.45-3.25$ & $7.2-10.9$ \\
\hline F3-4/ F4 & 2.0 & $>3.25$ & $>10.9$ \\
\hline
\end{tabular}

Table 1: Categorization of the degree of fibrosis based on cut off score of noninvasive markers.

Presence of hepatic steatosis using CAP measurement which is a novel method designed to determine the liver ultrasonic attenuation, with all measurements in the same area of liver parenchyma, expressed as $\mathrm{dB} / \mathrm{m}$. The final CAP corresponds to the median of individual CAP values (range 100 to $400 \mathrm{~dB} / \mathrm{m}$ ). Grading was done based on CAP score, S0 $<230 \mathrm{~dB} / \mathrm{m}, \mathrm{S} 1=230$ to $250 \mathrm{~dB} / \mathrm{m}, \mathrm{S} 2=251$ to $290 \mathrm{~dB} / \mathrm{m}, \mathrm{S} 3>290 \mathrm{~dB} / \mathrm{m}$ [35].

Presence of hepatic steatosis (HS) on USG. Qualitative grading of fatty liver was also recorded. If there was SO = no mention of fatty liver, S1 = mild fatty liver, S2 = moderate fatty liver, S3 = severe steatosis.

\section{Statistical analysis}

Qualitative data was represented in form of frequency and percentage. Qualitative data included sex, FIB 4 interpretation, APRI interpretation, HbeAg status, USG grade, Fibroscan interpretation, Fibroscan-CAP interpretation, AST/ALT ratio interpretation, etc. Association between qualitative variables was assessed by ChiSquare test with Continuity Correction for all 2 X 2 tables and with or without Continuity Correction in rest and Fisher's exact test for all 2 X 2 tables where p-value of Chi-Square test was not valid due to small counts. In presence of small counts in tables in more than two rows and/or columns, adjacent row and/or Column data was pooled and Chi-Square Test reapplied with Continuity Correction for all 2 X 2 tables and with or without Continuity Correction in rest and Fisher's Exact test for all 2 X 2 tables where p-value of ChiSquare test is not valid due to small counts in-spite of pooling of data (e.g. association between FIB-4 interpretation and Fibroscaninterpretation).

Quantitative data was represented using Mean \pm SD and Median and IQR (Interquartile range). Quantitative data included age, HBV viral load, FIB-4 values, APRI values, Fibroscan-E-score, FibroscanCAP score, Platelet count, AST, ALT and AST/ALT ratio. Correlation between Fibroscan-E-score and various variables was done using Pearson Correlation. Diagnostic efficacy of FIB-4 Interpretation, APRI interpretation and AST/ALT Ratio Interpretation with Fibroscan-interpretation as criterion was assessed by calculating sensitivity, specificity and AUROC. Binary Logistic Regression was applied to assess the predictive value of Fibroscan-CAP as independent (Predictor) variable for 'Fibroscan-interpretation' as dependent variable. Cohen's kappa statistic, $\kappa$, was used as measure of agreement between FIB 4 Interpretation, APRI interpretation and AST/ALT Ratio interpretation with Fibroscan interpretation as criterion. Kappa was interpreted on bases of cut-offs suggested by Landis and Koch, as follows:

\section{Kappa: Interpretation}

$<0$ : Poor agreement

0.0 - 0.20: Slight agreement

0.21 - 0.40: Fair agreement

0.41 - 0.60: Moderate agreement

0.61 - 0.80: Substantial agreement

0.81 - 1.00: Almost perfect agreement.

Appropriate statistical software, including but not restricted to MS Excel, PSPP version 0.8.5 was used for statistical analysis.

\section{Results}

A total of 188 patients with hepatitis B infection were identified, but 10 patients were excluded as per the exclusion criteria. The clinical and laboratory parameters of patients are shown in 
Role of Fibroscan and Non Invasive Markers to Assess Hepatic Fibrosis and Steatosis at Initial Presentation of Patients with Hepatitis B

table 2. There was a male predominance in the study with male to female ratio of the study patients was 2.1:1. The median age was 40 years (IQR $=24) .154$ patients $(86.5 \%)$ had asymptomatic hepatitis B infection. Remaining 24 patients (13.5\%) were symptomatic due to ascites, variceal bleeding or hepatocellular carcinoma. Amongst 100 patients who had normal AST and ALT, 40 patients had significant fibrosis (> F2) on fibroscan. Of the 139 patients who underwent fibroscan, 61 patients (44\%) had significant fibrosis. However, APRI, AST/ALT ratio and FIB4 detected significant fibrosis in 29 (16\%), 55 (31\%) and 52 (29\%) out of 178 patients.

\begin{tabular}{|l|c|}
\hline Parameter & Frequency \\
\hline Total number of patients referred & 188 \\
\hline Number of patients excluded & $10 / 188$ \\
\hline Male patients & $121 / 178$ \\
\hline Median age (years) & 40 \\
\hline Asymptomatic Chronic infection & $154 / 178$ \\
\hline Symptomatic due to decompensation of cirrhosis & $24 / 178$ \\
Ascites & 7 \\
Portal hypertension (variceal bleeding) & 11 \\
Hepatocellular carcinoma (HCC ) & 8 \\
\hline HBeAg Negative & $123 / 144$ \\
\hline HBV DNA viral load < 2000 IU/ml & $60 / 100$ \\
\hline $\begin{array}{l}\text { Significant fibrosis (>F2) along with normal AST } \\
\text { and ALT }\end{array}$ & $40 / 100$ \\
\hline
\end{tabular}

Table 2: Clinical and Laboratory parameters of patients.

Amongst the noninvasive biochemical tools for assessment of hepatic fibrosis (APRI, FIB-4 and AST/ALT ratio), only FIB-4 had significant correlation with fibroscan (Pearson correlation 0.65, p value $<0.05$ ) as shown in table 3 and figure 1 . Hepatic steatosis grades as defined by fibroscan-CAP score and ultrasound are shown in table 4.

The under ROC curve areas (AUROC) of FIB-4, APRI and AST/ ALT ratio that differentiated patients with significant hepatic fibrosis from those without were $0.704,0.674$ and 0.567 , respectively. The sensitivity and specificity of FIB-4, APRI and AST/ALT ratio to differentiate patients with significant hepatic fibrosis from those without was $42.6 \%$ and $92.3 \%, 72.1 \%$ and $60.2 \%, 29.5 \%$ and $83.3 \%$, respectively (Figure 2-4 and table 5). Although the sensitivity of APRI and FIB4 for detection of significant fibrosis is low, the specificity of FIB4 is better (92.3\%).

\begin{tabular}{|l|c|c|}
\hline Variables & & $\begin{array}{c}\text { Fibroscan } \\
\text { E-score }\end{array}$ \\
\hline Fibroscan-CAP score & Pearson Correlation & 0.077 \\
\hline & p-value & 0.367 \\
\hline FIB 4 & Pearson Correlation & $0.650\left(^{* *}\right)$ \\
\hline APRI & p-value & $4.82 E-18$ \\
\hline & Pearson Correlation & 0.107 \\
\hline AST/ALT Ratio & p-value & 0.211 \\
\hline \multicolumn{2}{|c|}{ Pearson Correlation } & 0.109 \\
\hline ** Correlation is significant at the 0.01 level (2-tailed). \\
\hline
\end{tabular}

Table 3: Correlation between laboratory tests and Fibroscan.

\begin{tabular}{|l|c|c|}
\hline Steatosis grades & Fibroscan - CAP (\%) & Ultrasound (\%) \\
\hline S0 & $59(42.4)$ & $108(61.4)$ \\
\hline S1 & $18(12.9)$ & $44(25)$ \\
\hline S2 & $34(24.5)$ & $20(11.4)$ \\
\hline S3 & $28(20.1)$ & $4(2.3)$ \\
\hline Total & $139(100)$ & $176(100)$ \\
\hline
\end{tabular}

Table 4: Hepatic Steatosis on Fibroscan (CAP score) and Ultrasound.

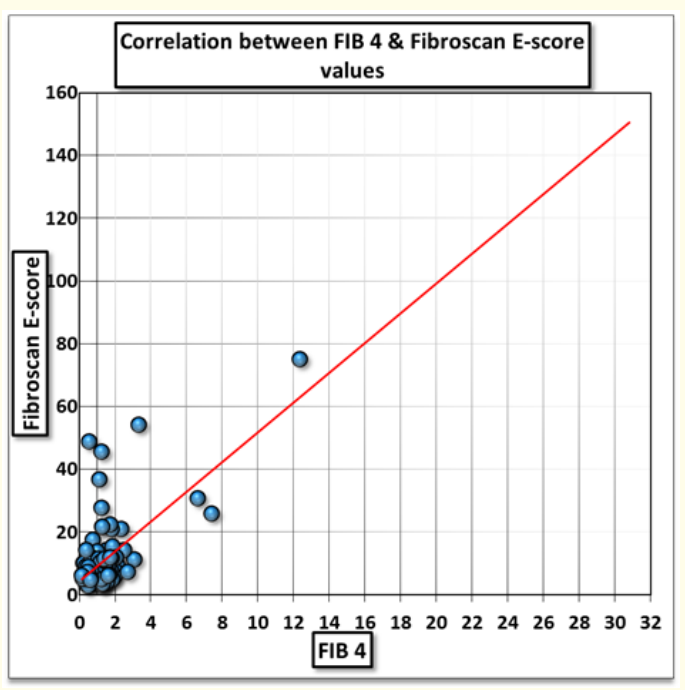

Figure 1: Scatter plot depicting correlation between FIB 4 and Fibroscan E-score. 


\begin{tabular}{|l|c|c|c|}
\hline Noninvasive test & APRI & FIB4 & AST/ALT ratio \\
\hline AUROC & 0.674 & 0.704 & 0.567 \\
\hline Associated criterion & $>0.26$ & $>1.42$ & $>1.04$ \\
\hline Sensitivity & 72.13 & 42.62 & 29.51 \\
\hline Specificity & 60.26 & 92.31 & 83.33 \\
\hline
\end{tabular}

Table 5: A comparison of the performance of each noninvasive test for the detection of significant fibrosis in patients with hepatitis B.

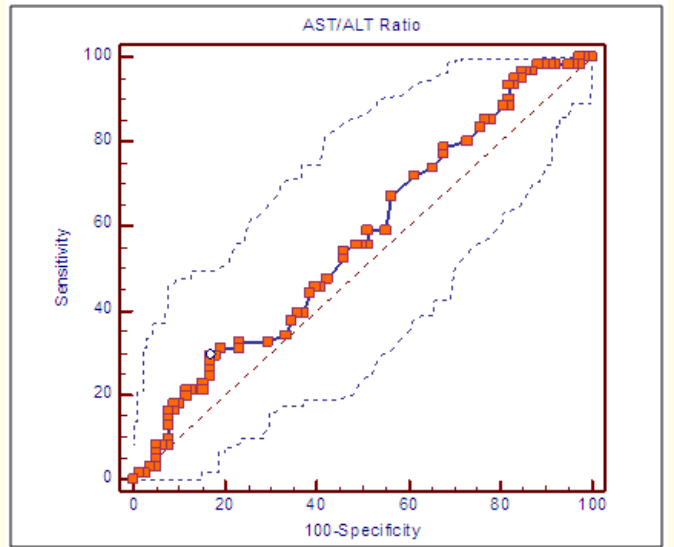

Figure 2: Receiver operating characteristic (ROC) curve of AST/ ALT ratio to detect significant fibrosis.

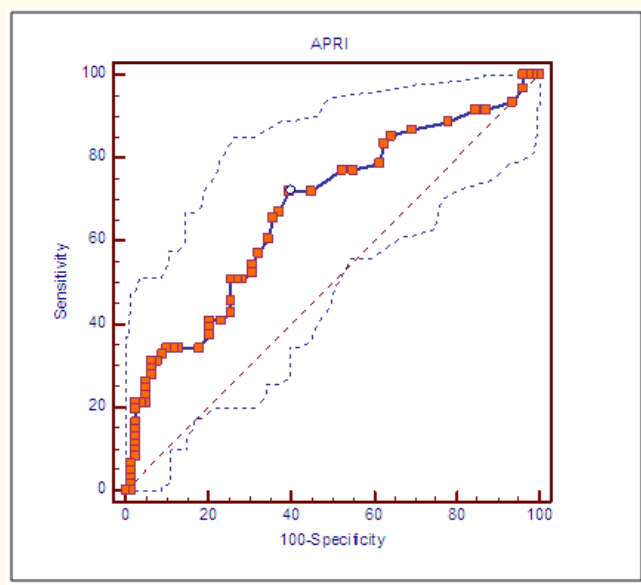

Figure 3: Receiver operating characteristic (ROC) curve of APRI to detect significant fibrosis.

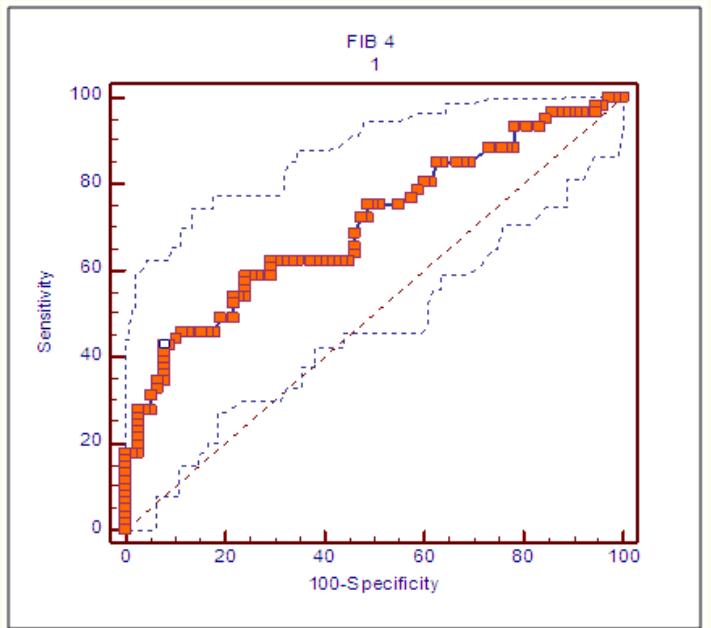

Figure 4: Receiver operating characteristic (ROC) curve of FIB4 to detect significant fibrosis.

After binary logistic regression analysis to assess the predictive value of Fibroscan-CAP as independent (Predictor) variable for 'Fibroscan-interpretation' as dependent variable, we did not find any significant association between both the variables.

\section{Discussion}

There was a male preponderance seen in this study as $70 \%$ of patients were males. This gender disparity has been shown in other studies [36]. The clinical characteristics including laboratory data findings were similar to other studies showing majority are HBeAg negative and have normal transaminases with our study showing $86.5 \%$ were HBeAg negative and $75 \%$ had normal liver enzymes [37].

In those with asymptomatic infection, approximately $17.7 \%$, $30 \%$ and $35 \%$ patients were found to have significant fibrosis at index presentation, using noninvasive tests such as APRI, FIB-4 and Fibroscan respectively. Using APRI alone as noninvasive tool for assessment of fibrosis may miss significant number of patients with significant fibrosis compared to FIB-4 and Fibroscan. However, all the markers including APRI (9.9\%), FIB-4 (9.9\%) and Fibroscan $(12.3 \%)$ detected similar rates of advanced fibrosis (F4). Classification of the degree of fibrosis using blood markers (APRI and FIB4) and APRI and Fibroscan was significantly different across the 2 investigative tools. However, there was no statistically significant 
difference in categorization of degree of fibrosis between FIB-4 and Fibroscan [14-16,22,23,25].

The group of patients who were found to have concomitant steatosis with Hepatitis B infection did not show a statistically significant difference in degree of fibrosis assessed by APRI, FIB- 4 and Fibroscan. The presence of hepatic steatosis can result in overestimation of the degree of fibrosis in these patients, however this was not seen in our study [7-11,38].

In patients with hepatitis $\mathrm{C}$, fat accumulation with hepatocytes can be associated with higher degree of fibrosis, however, fatty liver in association with CHB having similar correlation, still remains to be clarified [39].

Our study showed that concomitant HS was present in $61.5 \%$ patients using CAP as a screening tool. USG abdomen however, detected fatty liver in only $30 \%$ patients. Suggesting a significant difference in assessment of steatosis across both investigative modalities. However, many studies have also shown CAP to be superior to USG for detection of HS $[9,10]$.

Although previous studies have shown that approximately a quarter of patients with CHB have concomitant HS, with rising incidence of nonalcoholic fatty liver disease (NAFLD) the prevalence will continue to rise. Also, in these studies, USG was used as screening tool which has been found to be less sensitive compared to CAP.

\section{Limitation of the Study}

Liver biopsy was not done to evaluate fibrosis and hepatic steatosis. However, many studies and international guidelines have suggested good correlation between fibroscan and liver biopsy for assessment of liver fibrosis in chronic hepatitis B [17$19,22,25,26,31]$.

\section{Conclusion}

In conclusion, $\mathrm{CHB}$ is a silent infection which can progress to cirrhosis and its complications. Symptoms are present in advanced stage only, therefore early identification and assessment can prevent progression. Simple noninvasive tools can be used to identify patients with significant and advanced fibrosis and should be used in clinical practice to alert patients regarding the severity of underlying condition, as CHB being a silent infection is easily ignored. Using AST, ALT alone will miss significant fibrosis in large number of patients.

\section{Recommendation}

We recommend, that further studies evaluating the presence of risk factors such as obesity, diabetes, dyslipidemia for concomitant fatty liver in patients with $\mathrm{CHB}$, using combination of noninvasive tools along with liver biopsy will help to understand whether the relation of CHB with concomitant HS is casual or causal and to understand if presence of concomitant HS influences the degree of fibrosis.

Combination of these noninvasive tools can be used in outpatient clinic at initial assessment as, CHB being asymptomatic infection, majority of these patients will be reluctant to have liver biopsy. Use of these noninvasive tools, especially Fibroscan will help to improve compliance, and enhance timely evaluation of these patients. CAP might be more accurate than USG in detecting HS in patients with CHB.

\section{Source of Funding}

None.

\section{Conflicts of Interest}

All the authors have no conflict of interest.

\section{Bibliography}

1. Tandon BN., et al. "Epidemiology of Hepatitis B virus infection in India”. Gut 38 (1996): 56-59.

2. Ganem D and Prince AM. "Hepatitis B virus infection--natural history and clinical consequences". The New England Journal of Medicine 350 (2004): 1118-1129.

3. Iloeje UH., et al. "Natural history of chronic hepatitis B: what exactly has REVEAL revealed?" Liver International 32 (2012): 1333-1341.

4. Jang JW., et al. "Long-term effect of antiviral therapy on disease course after decompensation in patients with hepatitis B virus-related cirrhosis". Hepatology 61 (2015): 1809-1820.

5. Chon YE., et al. "Improvement of liver fibrosis after long-term antiviral therapy assessed by fibroscan in chronic hepatitis B patients with advanced fibrosis". American Journal of Gastroenterology 112 (2017): 882-891.

6. Kim JH., et al. "Clinical application of transient elastography in patients with chronic viral hepatitis receiving antiviral treatment". Liver International 35 (2015): 1103-1115. 
7. Bondini S., et al. "Impact of non-alcoholic fatty liver disease on chronic hepatitis B”. Liver International 27 (2007): 607-611.

8. Minakari M., et al. "Liver steatosis in patients with chronic hepatitis B infection: host and viral risk factors". European Journal of Gastroenterology and Hepatology 21 (2009): 512-516.

9. Cardoso AC., et al. "Diagnostic performance of controlled attenuation parameter for predicting steatosis grade in chronic hepatitis B". Annuals of Hepatology 14.6 (2015): 826-836.

10. Xu L., et al. "A comparison of hepatic steatosis index, controlled attenuation parameter and ultrasound as noninvasive diagnostic tools for steatosis in chronic hepatitis B". Digestive Liver Disease 49.8 (2017): 910-917.

11. Liang J., et al. "A Noninvasive Score Model for Prediction of NASH in Patients with Chronic Hepatitis B and Nonalcoholic Fatty Liver Disease". Biomed Research International (2017): 8793278.

12. Shiha G., et al. "Asian-Pacific Association for the Study of the Liver (APASL) consensus guidelines on invasive and non-invasive assessment of hepatic fibrosis: a 2016 update". Hepatology International 11.1 (2017): 1-30.

13. Lee S and Kim DY. "Non-invasive diagnosis of hepatitis B virus-related cirrhosis". World Journal of Gastroenterology 20.2 (2014): 445-459.

14. Xiao G., et al. "Comparison of diagnostic accuracy of aspartate aminotransferase to platelet ratio index and fibrosis-4 index for detecting liver fibrosis in adult patients with chronic hepatitis B virus infection: a systemic review and meta-analysis". Hepatology 61.1 (2015): 292-302.

15. Shin WG., et al. "Aspartate aminotransferase to platelet ratio index (APRI) can predict liver fibrosis in chronic hepatitis B". Digestive Liver Disease 40 (2008): 267-274.

16. Kim BK., et al. "Noninvasive models to predict liver cirrhosis in patients with chronic hepatitis B". Liver International 27.7 (2007): 969-976.

17. Singh S., et al. "American Gastroenterological Association Institute Technical Review on the Role of Elastography in Chronic Liver Diseases". Gastroenterology 152.6 (2017): 1544-1577.

18. Sharma P., et al. "Usefulness of transient elastography by FibroScan for the evaluation of liver fibrosis". Indian Journal of Gastroenterology 33.5 (2014): 445-451.
19. Afdhal NH., et al. "Accuracy of fibroscan, compared with histology, in analysis of liver fibrosis in patients with hepatitis B or C: a United States multicenter study". Clinical Gastroenterology and Hepatology 13.4 (2015): 772-9.e1-e3.

20. Bota S., et al. "Meta-analysis: ARFI elastography versus transient elastography for the evaluation of liver fibrosis". Liver International 33.8 (2013): 1138-1147.

21. Lee GM., et al. "Quantitative Measurement of Hepatic Fibrosis with Gadoxetic Acid-Enhanced Magnetic Resonance Imaging in Patients with Chronic Hepatitis B Infection: A Comparative Study on Aspartate Aminotransferase to Platelet Ratio Index and Fibrosis-4 Index". Korean Journal of Radiology 18.3 (2017): 444-451.

22. Li Y., et al. "Development of algorithms based on serum markers and transient elastography for detecting significant fibrosis and cirrhosis in chronic hepatitis B patients: Significant reduction in liver biopsy". Hepatol Res. 46.13 (2016): 13671379.

23. Ding D., et al. "FibroScan, aspartate aminotransferase and alanine aminotransferase ratio (AAR), aspartate aminotransferase to platelet ratio index (APRI), fibrosis index based on the 4 factor (FIB-4), and their combinations in the assessment of liver fibrosis in patients with hepatitis B". International Journal of Clinical and Experimental Medicine 8.11 (2015): 20876-82.

24. Jia J., et al. "Transient elastography compared to serum markers to predict liver fibrosis in a cohort of Chinese patients with chronic hepatitis B". Journal of Gastroenterology Hepatology 30.4 (2015): 756-762.

25. Chang PE., et al. "Prospective evaluation of transient elastography for the diagnosis of hepatic fibrosis in Asians: comparison with liver biopsy and aspartate transaminase platelet ratio index". Alimentary Pharmacology and Therapeutics 28.1 (2008): 51-61.

26. Cheng J., et al. "Validation of Ten Noninvasive Diagnostic Models for Prediction of Liver Fibrosis in Patients with Chronic Hepatitis B". PLoS One 10.12 (2015): e0144425.

27. Ratziu V., et al. "Sampling variability of liver biopsy in nonalcoholic fatty liver disease". Gastroenterology 128.7 (2005): 1898-1906.

28. Guido M., et al. "Chronic viral hepatitis: the histology report". Digestive Liver Disease 43 (2011): S331-343. 
29. Marcellin P., et al. "Non-invasive assessment of liver fibrosis by stiffness measurement in patients with chronic hepatitis B". Liver International 29.2 (2009): 242-247.

30. Chan HL., et al. "Alanine aminotransferase-based algorithms of liver stiffness measurement by transient elastography (Fibroscan) for liver fibrosis in chronic hepatitis B". Journal of Viral Hepatitis 16.1 (2009): 36-44.

31. European Association for Study of Liver Asociacion Latinoamericana para el Estudio del Higado. "EASL-ALEH Clinical Practice Guidelines: Non-invasive tests for evaluation of liver disease severity and prognosis". Journal of Hepatology 63.1 (2015): 237-264.

32. Kwo PY., et al. "ACG Clinical Guideline: Evaluation of Abnormal Liver Chemistries". American Journal of Gastroenterology 112.1 (2017): 18-35.

33. Sandrin L., et al. "Shear elasticity probe for soft tissues with 1-D transient elastography". IEEE Transactions on Ultrasonics, Ferroelectrics, and Frequency Control 49.4 (2002): 436-446.

34. Cai YJ., et al. "A diagnostic algorithm for assessment of liver fibrosis by liver stiffness measurement in patients with chronic hepatitis B". Journal of Viral Hepatitis (2017).

35. Wang Y., et al. "Controlled attenuation parameter for assessment of hepatic steatosis grades: a diagnostic meta-analysis". International Journal of Clinical and Experimental Medicine 8.10 (2015): 17654-17663.

36. Baig S. "Gender disparity in infections of Hepatitis B virus". Journal of College of Physicians and Surgeons Pakistan 19.9 (2009): 598-600.

37. Guardiola Arévalo A., et al. "Characteristics and course of chronic hepatitis B e antigen-negative infection". Gastroenterology Hepatology 40.2 (2017): 59-69.

38. Lim CT and Kumar R. "Hepatitis B and concomitant hepatic steatosis”. Annals of Translational Medicine's 5.3 (2017): 38.

39. Fan JG and Chitturi S. "Hepatitis B and fatty liver: causal or coincidental?" Journal of Gastroenterology Hepatology 23.5 (2008): 679-681.

\section{Assets from publication with us}

- Prompt Acknowledgement after receiving the article

- Thorough Double blinded peer review

- Rapid Publication

- Issue of Publication Certificate

- High visibility of your Published work

Website: www.actascientific.com/

Submit Article: www.actascientific.com/submission.php

Email us: editor@actascientific.com

Contact uS: +919182824667

Citation: Shah Jayshri A., et al. "Role of Fibroscan and Non Invasive Markers to Assess Hepatic Fibrosis and Steatosis at Initial Presentation of Patients with Hepatitis B". Acta Scientific Gastrointestinal Disorders 3.8 (2020): 16-23. 